\title{
A THEOREM OF LIOUVILLE TYPE FOR $p$-HARMONIC MAPS IN WEIGHTED RIEMANNIAN MANIFOLDS
}

\author{
JiAN-Feng Zhang AND Yue Wang
}

\begin{abstract}
Let $M$ be a weighted Riemannian manifold with non-negative Bakry-Émery-Ricci curvature and $N$ be a complete Riemannian manifold of non-positive sectional curvature. In this paper, the $p$-harmonic map $u: M \rightarrow N$ is studied, and a theorem of Liouville type is obtained.
\end{abstract}

\section{Introduction}

Let $\left(M^{m}, g\right)$ and $\left(N^{n}, h\right)$ be complete Riemannian manifolds, $\operatorname{dim} M=$ $m \geq 2, \operatorname{dim} N=n$, and let $p \geq 2$. A map $u: M \rightarrow N$ is said to be $p$-harmonic if $\left.u\right|_{\Omega}$ is a critical point of the $p$-energy

$$
E_{p}(u)=\frac{1}{p} \int_{\Omega}|d u|^{p} d V_{M},
$$

for every compact domain $\Omega \subset M$. Here the differential $d u$ is a section of the bundle $T^{*} M \otimes u^{-1} T N \rightarrow M$ and $u^{-1} T N$ denotes the pull-back bundle via the map $u$ and $d V_{M}$ stands for the canonical Riemannian volume form on $M$. When $u$ is $C^{2}$-regular, the Euler-Lagrange equation for the energy functional $E_{p}$ is the $p$-harmonic maps equation [2]

$$
\tau_{p}(u):=\operatorname{div}\left(|d u|^{p-2} d u\right)=|d u|^{p-2} \tau_{2}(u)+(p-2)|d u|^{p-3} d u\left(\operatorname{grad}_{g}|d u|\right)=0
$$

where $\tau_{2}(u):=\operatorname{div}(d u)$ is the standard tension field of $u$. In this paper, $\triangle, \delta$ and $\tau(u)=\tau_{2}(u)$ always denote the Laplace operator, the co-differential operator and the tension field of a map $u$ on the manifold $\left(M^{m}, g\right)$. Several studies are given for harmonic maps (see $[5,7,11,13,14])$. For these harmonic maps, there

2010 Mathematics Subject Classification. 53C20.

Key words and phrases. Weighted Riemannian manifolds; $p$-harmonic map; $f$-harmonic map; Bakry-Émery-Ricci curvature; liouville type theorem.

This article was supported in part by National Natural Science Foundation of China (No. 10901147), Project of Lishui High-level Talents (2014RC29) and Professional development program for domestic visiting scholars in Universities (FX2013149).

Received April 28, 2015; revised October 13, 2015. 
are Liouville type theorems, which state that a harmonic map $u$ is constant under some conditions.

In 1976, R. M. Schoen and S. T. Yau [11] proved the following Liouville type theorem.

THEOREM 1.1. Let $M$ be a complete Riemannian manifold of non-negative Ricci curvature and $N$ be a complete Riemannian manifold of non-positive sectional curvature. Then any harmonic map $u: M \rightarrow N$ of $E_{2}(u)<\infty$ is constant. 1998.

For $p$-harmonic maps, N. Nakauchi [9] proved the following theorem in

THEOREM 1.2. Let $M$ be a complete Riemannian manifold of non-negative Ricci curvature and $N$ be a complete Riemannian manifold of non-positive sectional curvature. Then any p-harmonic map $u: M \rightarrow N$ of $E_{p}(u)<\infty$ is constant.

Let $f: M \rightarrow R$ be a smooth function. A map $u: M \rightarrow N$ is said to be (weakly) $f$-harmonic if $\left.u\right|_{\Omega}$ is a critical point of the $f$-energy

$$
E_{f}(u)=\frac{1}{2} \int e^{-f}|d u|^{2} d V_{M}
$$

for every compact domain $\Omega \subset M$. The study of $f$-harmonic maps began with A. Lichnerowicz in 1969 [6] and J. Eells and L. Lemaire in 1977 [3]. We now study the $f$-harmonic maps on weighted manifold and gradient Ricci solitons.

A weighted manifold, also known in the literature as smooth metric measure space, is a Riemannian manifold $\left(M^{m}, g\right)$ endowed with a weighted volume form $e^{-f} d V_{M}$ and some smooth function $f: M \rightarrow R$. For a weighted manifold $\left(M^{m}, g, e^{-f} d V_{M}\right)$, we are interested in the Bakry-Émery Ricci tensor $\operatorname{Ric}_{f}^{M}=$ $\mathrm{Ric}^{M}+$ Hess $f$, which was first introduced by A. Lichnerowicz in [7] and later by D. Bakry and M. Émery in [1]. Recently it has been found that this curvature tensor is strictly related with geometric objects whose importance is outstanding in mathematics. Imposing the constancy of $\mathrm{Ric}_{f}^{M}$, one can introduce gradient Ricci soliton structure on the manifold, and the importance of gradient Ricci solitons is due to the fact that they correspond to self-similar solutions to Hamilton's Ricci flow and often arise as limits of dilations of singularities developed along the flow.

It is easy to know that the $f$-harmonic map on manifold $\left(M^{m}, g\right)$, just be the harmonic map on a weighted manifold $\left(M^{m}, g, e^{-f} d V_{M}\right)$. In this paper, we study the $p$-harmonic maps on a weighted manifold $\left(M^{m}, g, e^{-f} d V_{M}\right)$, that is, the map is a critical point of the $(p, f)$-energy

$$
E_{p, f}(u)=\frac{1}{p} \int_{\Omega} e^{-f}|d u|^{p} d V_{M}
$$

for every compact domain $\Omega \subset M$. We said $u$ is a $(p, f)$-harmonic map on $M$. We obtain the following general result. 
THEOREM 1.3. Let $\left(M^{m}, g, e^{-f} d V_{M}\right)$ be an orientable, complete non-compact weighted Riemannian manifold with $\operatorname{Ric}_{f}^{M} \geq 0$, and $N$ be a complete Riemannian manifold of non-positive sectional curvature, where $f \in C^{\infty}(M)$. Let $u: M \rightarrow N$ be a $(p, f)$-harmonic map with $E_{p, f}(u)<\infty$.

(I) Assume at least one of the following assumption is satisfied

(a) there exists a constant $C>0$ such that $|f| \leq C$;

(b) $f$ is convex and the set of its critical points is unbounded;

(c) $\operatorname{Vol}_{f}(M):=\int_{M} e^{-f} d V_{M}=+\infty$;

(d) there is a point $q_{0} \in M$ such that $\left.\operatorname{Ric}_{f}^{M}\right|_{q_{0}}>0$

(e) there is a point $q_{1} \in M$ such that $\left.\operatorname{Ric}^{M}(X, X)\right|_{q_{1}} \neq 0$ for all $0 \neq X \in T_{q_{1}} M$.

Then $u$ is homotopic to a constant.

(II) If $\operatorname{Sect}^{N}<0$, then $u$ is homotopic either to a constant or to a totally geodesic map whose image is contained in a geodesic of $N$.

\section{Bochner type formula}

Let $\left(M^{m}, g, e^{-f} d V_{M}\right)$ be a weighted manifold, or a smooth metric measure space, where $f: M \rightarrow R$ is a smooth function. For every smooth function $h$ on $M$, we define an operator $L_{f}$ as follows:

$$
L_{f}(h)=\triangle h-\langle d f, d h\rangle,
$$

where $\triangle=-(d \delta+\delta d)$ is the Laplace operator. At the same time, the BakryÉmery-Ricci curvature is defined by the formula

$$
\operatorname{Ric}_{f}^{M}=\operatorname{Ric}^{M}+\text { Hess } f \text {. }
$$

LeMma 2.1. Let $\left(M^{m}, g, e^{-f} d V_{M}\right)$ be a weighted Riemannian manifold and $N$ be a smooth Riemannian manifold, then a map $u: M \rightarrow N$ is $(p, f)$-harmonic if and only if it satisfies the Euler-Lagrange equation

$$
\begin{aligned}
\tau_{p, f}(u) & :=e^{-f}|d u|^{p-2}\left(\tau(u)+d u\left(\operatorname{grad}\left(\ln |d u|^{p-2}-f\right)\right)\right. \\
& =\operatorname{div}\left(e^{-f}|d u|^{p-2} d u\right)=-\delta\left(e^{-f}|d u|^{p-2} d u\right)=0
\end{aligned}
$$

where $\tau(u)=-\delta d u=\operatorname{div}(d u)$ is the tension field of $u . \quad$ We call $\tau_{p, f}(u)$ the $(p, f)$ tension field of $u$.

Proof. The proof is standard computation which can be adapted from the case when $p=2$ and $f=0$, see for example [3].

In this section we give the following Bochner type formula.

LeMma 2.2. Let $\left(M^{m}, g, e^{-f} d V_{M}\right)$ be a weighted Riemannian manifold and $N$ be a smooth Riemannian manifold, and $u: M \rightarrow N$ be a $C^{2}$ map. Then 


$$
\begin{aligned}
L_{f}\left(\frac{1}{p}|d u|^{p}\right)= & |d u|^{p-2}\left(|\nabla d u|^{2}+(p-2)|\nabla| d u||^{2}+F_{f}(u)\right) \\
& +|d u|^{p-2}\langle d(\tau(u)-d u(\nabla f)), d u\rangle
\end{aligned}
$$

where

$$
F_{f}(u)=\sum\left\langle d u\left(\operatorname{Ric}_{f}^{M} e_{i}\right), d u\left(e_{i}\right)\right\rangle-\sum\left\langle R^{N}\left(d u\left(e_{i}\right), d u\left(e_{j}\right)\right) d u\left(e_{i}\right), d u\left(e_{j}\right)\right\rangle .
$$

Proof. We start recalling the standard Bochner formula for a smooth map $u[3]$,

$$
\frac{1}{2} \triangle|d u|^{2}=\langle\triangle d u, d u\rangle+|\nabla d u|^{2}+F(u)
$$

where

$$
F(u)=\sum\left\langle d u\left(\operatorname{Ric}^{M} e_{i}\right), d u\left(e_{i}\right)\right\rangle-\sum\left\langle R^{N}\left(d u\left(e_{i}\right), d u\left(e_{j}\right)\right) d u\left(e_{i}\right), d u\left(e_{j}\right)\right\rangle .
$$

Then

$$
\begin{aligned}
L_{f}\left(\frac{1}{p}|d u|^{p}\right)= & \frac{1}{p} \triangle|d u|^{p}-\left\langle d f, d\left(\frac{1}{p}|d u|^{p}\right)\right\rangle \\
= & \frac{1}{2}|d u|^{p-2} \triangle|d u|^{2}+(p-2)|d u|^{p-2}|\nabla| d u||^{2}-\frac{1}{2}|d u|^{p-2}\left\langle d f, d\left(|d u|^{2}\right)\right\rangle \\
= & |d u|^{p-2}\left(\langle\triangle d u, d u\rangle+|\nabla d u|^{2}+F(u)\right) \\
& +(p-2)|d u|^{p-2}|\nabla| d u||^{2}-\frac{1}{2}|d u|^{p-2}\left\langle d f, d\left(|d u|^{2}\right)\right\rangle \\
= & |d u|^{p-2}\left(|\nabla d u|^{2}+(p-2)|\nabla| d u||^{2}+F_{f}(u)\right) \\
& +|d u|^{p-2}\left(\langle\triangle d u, d u\rangle-\left\langle d u\left(\nabla_{(.)} \nabla f\right), d u\right\rangle\right)-\frac{1}{2}|d u|^{p-2}\left\langle d f, d\left(|d u|^{2}\right)\right\rangle
\end{aligned}
$$

where

$$
\begin{aligned}
F_{f}(u) & =\sum\left\langle d u\left(\operatorname{Ric}_{f}^{M} e_{i}\right), d u\left(e_{i}\right)\right\rangle-\sum\left\langle R^{N}\left(d u\left(e_{i}\right), d u\left(e_{j}\right)\right) d u\left(e_{i}\right), d u\left(e_{j}\right)\right\rangle . \\
& =F(u)+\sum\left\langle d u\left(\nabla_{e_{i}} \nabla f\right), d u\left(e_{i}\right)\right\rangle \\
& =F(u)+\left\langle d u\left(\nabla_{(.)} \nabla f\right), d u\right\rangle .
\end{aligned}
$$

It is easy to know that

$$
\triangle d u=-(d \delta+\delta d) d u=-d(\delta d) u=d(\tau(u)) .
$$

Hence the lemma is proved once we show that

$$
\left.\left\langle d u\left(\nabla_{(.)} \nabla f\right), d u\right\rangle\right)+\frac{1}{2}\left\langle d f, d\left(|d u|^{2}\right)\right\rangle=\langle d(d u(\nabla f)), d u\rangle .
$$


Let $\left\{x_{a}\right\}_{a=1}^{m}$ be the normal coordinate chart at $q \in M$ on $M$ and $\left\{\theta_{A}\right\}_{A=1}^{n}$ and $\left\{E_{A}\right\}_{A=1}^{n}$ orthonormal coframe and dual frame on $N$ at $u(q)$ respectively. Moreover denote the components of the metric on $M$ as $g_{a b}:=g_{M}\left(\frac{\partial}{\partial x^{a}}, \frac{\partial}{\partial x^{b}}\right)$.
Then we have the following equalities in this coordinate.

$$
g_{a b}=g^{a b}=\left\{\begin{array}{ll}
1 & (a=b) \\
0 & (a \neq b)
\end{array}, \quad \frac{\partial g^{a b}}{\partial x^{c}}=0, \quad \Gamma_{a b}^{c}=0 \quad \text { at } q .\right.
$$

Now, we can write

$$
\begin{gathered}
d u=u_{a}^{A} E_{A} \otimes d x^{a}, \quad \nabla f=f^{a} \frac{\partial}{\partial x^{a}} \\
\nabla_{\partial / \partial x^{a}} \nabla f=\nabla_{\partial / \partial x^{a}}\left(f^{b} \frac{\partial}{\partial x^{b}}\right)=\left(f_{a}^{c}+f^{b} \Gamma_{a b}^{c}\right) \frac{\partial}{\partial x^{c}}
\end{gathered}
$$

that is,

$$
\nabla_{(.)} \nabla f=\left(f_{a}^{c}+f^{b} \Gamma_{a b}^{c}\right) d x^{a} \otimes \frac{\partial}{\partial x^{c}} .
$$

At the given point $q \in M$, we have

$$
\begin{gathered}
d u\left(\nabla_{(.)} \nabla f\right)=u_{c}^{A} f_{a}^{c} d x^{a} \otimes E_{A} . \\
\left\langle d u\left(\nabla_{(.)} \nabla f\right), d u\right\rangle=g^{a d} u_{c}^{A} u_{d}^{A} f_{a}^{c}=u_{c}^{A} u_{a}^{A} f_{a}^{c} .
\end{gathered}
$$

and

$$
\left\langle d f, d\left(|d u|^{2}\right)\right\rangle=\left\langle f_{a} d x^{a}, \frac{\partial}{\partial x^{d}}\left(g^{b c} u_{c}^{A} u_{b}^{A}\right) d x^{d}\right\rangle=2 f^{a} u_{b}^{A} u_{b a}^{A}
$$

Then

$$
\left\langle d u\left(\nabla_{(.)} \nabla f\right), d u\right\rangle+\frac{1}{2}\left\langle d f, d\left(|d u|^{2}\right)\right\rangle=u_{c}^{A} u_{a}^{A} f_{c}^{a}+f^{a} u_{b}^{A} u_{b a}^{A}
$$

On the other hand,

$$
\begin{aligned}
\langle d u, d(d u(\nabla f))\rangle= & \left\langle d u, d\left(f^{c} u_{c}^{A} E_{A}\right)\right. \\
= & \left\langle d u, \frac{\partial}{\partial x^{a}}\left(f^{c} u_{c}^{A} E_{A}\right) \otimes d x^{a}\right\rangle \\
= & \left\langle u_{d}^{A} d x^{d} \otimes E_{A}, u_{c}^{A} f_{a}^{c} d x^{a} \otimes E_{A}\right\rangle \\
& +\left\langle u_{c}^{A} d x^{c} \otimes E_{A}, u_{b a}^{A} f^{a} d x^{b} \otimes E_{A}\right\rangle \\
= & u_{c}^{A} u_{a}^{A} f_{c}^{a}+f^{a} u_{b}^{A} u_{b a}^{A} .
\end{aligned}
$$

This latter proves (2.3) and concludes the proof. 


\section{The proof of theorem $\mathbf{1 . 3}$}

Proof of theorem 1.3. The (2.1) can be rewritten

$$
\begin{aligned}
\frac{1}{2} L_{f}\left(|d u|^{p}\right)= & \frac{p}{2}|d u|^{p-2}\left(|\nabla d u|^{2}+(p-2)|\nabla| d u||^{2}+F_{f}(u)\right) \\
& +\frac{p}{2}|d u|^{p-2}\langle d(\tau(u)-d u(\nabla f)), d u\rangle
\end{aligned}
$$

Let $\phi=|d u|^{p / 2}$, then

$$
\frac{1}{2} L_{f}\left(\phi^{2}\right)=\phi L_{f}(\phi)+|\nabla \phi|^{2}
$$

It is easy to get

$$
|\nabla \phi|^{2}=\frac{p^{2}}{4}|d u|^{p-2}|\nabla| d u||^{2}
$$

Using the Kato's inequality, we have

$$
\begin{aligned}
\phi L_{f}(\phi)= & \frac{1}{2} L_{f}\left(\phi^{2}\right)-|\nabla \phi|^{2} \\
= & \frac{1}{2} L_{f}\left(|d u|^{p}\right)-\frac{p^{2}}{4}|d u|^{p-2}|\nabla| d u||^{2} \\
= & \frac{p}{2}|d u|^{p-2}\left(|\nabla d u|^{2}+(p-2)|\nabla| d u||^{2}+F_{f}(u)\right) \\
& +\frac{p}{2}|d u|^{p-2}\langle d(\tau(u)-d u(\nabla f)), d u\rangle-\frac{p^{2}}{4}|d u|^{p-2}|\nabla| d u||^{2} \\
= & \frac{p}{4}|d u|^{p-2}\left(2|\nabla d u|^{2}+(p-4)|\nabla| d u||^{2}+2 F_{f}(u)\right) \\
& +\frac{p}{2}|d u|^{p-2}\langle d(\tau(u)-d u(\nabla f)), d u\rangle \\
\geq & \frac{p}{4}|d u|^{p-2}\left((p-2)|\nabla| d u||^{2}+2 F_{f}(u)\right) \\
& +\frac{p}{2}|d u|^{p-2}\langle d(\tau(u)-d u(\nabla f)), d u\rangle
\end{aligned}
$$

Let $B(r)$ be a ball with radius $r$, and $w_{r}$ be a cut-off function s.t. $w_{r} \leq 1$ on $M$, $\left.w_{r}\right|_{B(r)} \equiv 1,\left.w_{r}\right|_{M \backslash B(2 r)} \equiv 0$ and $\left|\nabla w_{r}\right| \leq \frac{2}{r}$. Since $F_{f}(u) \geq 0$, we have

$$
\begin{gathered}
\frac{p}{2} \int_{M} w_{r}^{2}|d u|^{p-2}(\langle d(\tau(u)-d u(\nabla f)) d u\rangle) e^{-f} d v_{g}-\int_{M} w_{r}^{2} \phi L_{f}(\phi) e^{-f} d v_{g} \\
\leq-\frac{p}{4} \int_{M} w_{r}^{2}|d u|^{p-2}\left((p-2)|\nabla| d u||^{2}+2 F_{f}(u)\right) e^{-f} d v_{g} \leq 0
\end{gathered}
$$


where $d v_{g}$ stands for the canonical Riemannian volume form on metric $g$. It is easy to know that

$$
\begin{aligned}
\int_{M} w_{r}^{2} \phi L_{f}(\phi) e^{-f} d v_{g} \\
\quad=\int_{M} w_{r}^{2} \phi \operatorname{div}\left(e^{-f} d(\phi)\right) d v_{g} \\
\quad=\int_{M} w_{r}^{2} \phi\left(-\delta\left(e^{-f} d(\phi)\right)\right) d v_{g} \\
=-\int_{M}\left\langle d\left(w_{r}^{2} \phi\right), e^{-f} d(\phi)\right\rangle d v_{g} \\
=-\int_{M} w_{r}^{2} e^{-f}|d(\phi)|^{2} d v_{g}-2 \int_{M}\left\langle w_{r} d\left(w_{r}\right) \phi, e^{-f} d(\phi)\right\rangle d v_{g} \\
\leq-\int_{M} w_{r}^{2} e^{-f}|d(\phi)|^{2} d v_{g}+2 \int_{M} w_{r}\left|d\left(w_{r}\right)\right| \phi|d(\phi)| e^{-f} d v_{g} \\
=-\int_{M} w_{r}^{2} e^{-f}|d(\phi)|^{2} d v_{g}+p \int_{M} w_{r}\left|d\left(w_{r}\right)\right||d u|^{p-1}|d(|d u|)| e^{-f} d v_{g}
\end{aligned}
$$

At the same time, we have

$$
\begin{aligned}
& \frac{p}{2} \int_{M} w_{r}^{2}|d u|^{p-2}(\langle d(\tau(u)-d u(\nabla f)), d u\rangle) e^{-f} d v_{g} \\
&=\frac{p}{2} \int_{M}\left\langle d(\tau(u)-d u(\nabla f)), w_{r}^{2} e^{-f}|d u|^{p-2} d u\right\rangle d v_{g} \\
&=\frac{p}{2} \int_{M}\left\langle\tau(u)-d u(\nabla f), \delta\left(w_{r}^{2} e^{-f}|d u|^{p-2} d u\right)\right\rangle d v_{g} \\
&=\frac{p}{2} \int_{M}\left\langle\tau(u)-d u(\nabla f), w_{r}^{2} \delta\left(e^{-f}|d u|^{p-2} d u\right)+e^{-f}|d u|^{p-2} d u\left(\nabla w_{r}^{2}\right)\right\rangle d v_{g} \\
&=\frac{p}{2} \int_{M}\left\langle\tau(u)-d u(\nabla f), e^{-f}|d u|^{p-2} d u\left(\nabla w_{r}^{2}\right)\right\rangle d v_{g} \\
&\left(\delta\left(e^{-f}|d u|^{p-2} d u\right)=-\tau_{p, f}(u)=0\right) \\
&= \frac{p}{2} \int_{M}\left\langle e^{-f}(\tau(u)-d u(\nabla f)),|d u|^{p-2} d u\left(\nabla w_{r}^{2}\right)\right\rangle d v_{g} \\
&= \frac{p}{2} \int_{M}\left\langle-\delta\left(e^{-f} d u\right),|d u|^{p-2} d u\left(\nabla w_{r}^{2}\right)\right\rangle d v_{g} \\
&=-\frac{p}{2} \int_{M}\left\langle e^{-f} d u, d\left(|d u|^{p-2} d u\left(\nabla w_{r}^{2}\right)\right)\right\rangle d v_{g}
\end{aligned}
$$




$$
\begin{aligned}
& =-\frac{p}{2}\left(\int_{M}\left\langle e^{-f} d u,(p-2)|d u|^{p-3} d(|d u|) d u\left(\nabla w_{r}^{2}\right)\right\rangle d v_{g}\right. \\
& \left.\quad+\int_{M}\left\langle e^{-f} d u,|d u|^{p-2} d\left(d u\left(\nabla w_{r}^{2}\right)\right)\right\rangle d v_{g}\right) \\
& =-\frac{p}{2}\left(\int_{M}\left\langle e^{-f} d u,(p-2)|d u|^{p-3} d(|d u|) d u\left(\nabla w_{r}^{2}\right)\right\rangle d v_{g}\right. \\
& \left.\quad+\int_{M}\left\langle e^{-f}|d u|^{p-2} d u, d\left(d u\left(\nabla w_{r}^{2}\right)\right)\right\rangle d v_{g}\right) \\
& =-\frac{p}{2}\left(\int_{M}\left\langle e^{-f} d u,(p-2)|d u|^{p-3} d(|d u|) d u\left(\nabla w_{r}^{2}\right)\right\rangle d v_{g}\right. \\
& \left.\quad+\int_{M}\left\langle\delta\left(e^{-f}|d u|^{p-2} d u\right), d u\left(\nabla w_{r}^{2}\right)\right\rangle d v_{g}\right) \\
& =-\frac{p}{2} \int_{M}\left\langle e^{-f} d u,(p-2)|d u|^{p-3} d(|d u|) d u\left(\nabla w_{r}^{2}\right)\right\rangle d v_{g} \\
& \geq-p(p-2) \int_{M} w_{r}\left|d\left(w_{r}\right)\right||d u|^{p-1}|d(|d u|)| e^{-f} d v_{g}
\end{aligned}
$$

Here we have used the fact $\delta\left(e^{-f}|d u|^{p-2} d u\right)=-\tau_{p, f}(u)=0$. Now, we have

$$
\begin{aligned}
0 & \geq \frac{p}{2} \int_{M} w_{r}^{2}|d u|^{p-2}(\langle d(\Delta u-d u(\nabla f)) d u\rangle) e^{-f} d v_{g}-\int_{M} w_{r}^{2} \phi L_{f}(\phi) e^{-f} d v_{g} \\
& \geq \int_{M} w_{r}^{2} e^{-f}|d(\phi)|^{2} d v_{g}-p(p-1) \int_{M} w_{r}\left|d\left(w_{r}\right)\right||d u|^{p-1}|d(|d u|)| e^{-f} d v_{g} \\
& \geq \int_{M} w_{r}^{2} e^{-f}|d(\phi)|^{2} d v_{g}-2(p-1) \int_{M} w_{r}\left|d\left(w_{r}\right)\right| \phi|d(\phi)| e^{-f} d v_{g} \\
& \geq(1-(p-1) \varepsilon) \int_{M} w_{r}^{2} e^{-f}|d(\phi)|^{2} d v_{g}-\frac{(p-1)}{\varepsilon} \int_{M}\left|d\left(w_{r}\right)\right|^{2} \phi^{2} e^{-f} d v_{g}
\end{aligned}
$$

for any $0<\varepsilon<1$. So $d(\phi) \in L^{2}$. Hence by the Hölder inequality

$$
\begin{aligned}
\int_{\Omega}|d u|^{p-1}|d(|d u|)| e^{-f} d v_{g} & =\frac{2}{p} \int_{\Omega} \phi|d(\phi)| e^{-f} d v_{g} \\
& \leq \frac{2}{p}\left(\int_{\Omega} e^{-f}|d(\phi)|^{2} d v_{g}\right)^{1 / 2}\left(\int_{\Omega} \phi^{2} e^{-f} d v_{g}\right)^{1 / 2}<\infty
\end{aligned}
$$

for any compact set $\Omega \subset M$. If we let $r \rightarrow \infty$, then

$$
\int_{M} w_{r}\left|d\left(w_{r}\right)\right||d u|^{p-1}|d(|d u|)| e^{-f} d v_{g} \rightarrow 0 .
$$


From above we get

$$
\begin{aligned}
0 & \geq-\frac{p}{4} \int_{M} w_{r}^{2}|d u|^{p-2}\left((p-2)|\nabla| d u||^{2}+2 F_{f}(u)\right) e^{-f} d v_{g} \\
& =\frac{p}{2} \int_{M} w_{r}^{2}|d u|^{p-2}(\langle d(\triangle u-d u(\nabla f)) d u\rangle) e^{-f} d v_{g}-\int_{M} w_{r}^{2} \phi L_{f}(\phi) e^{-f} d v_{g} \\
& \geq \int_{M} w_{r}^{2} e^{-f}|d(\phi)|^{2} d v_{g}
\end{aligned}
$$

That is

$$
|d u|^{p-2}\left((p-2)|\nabla| d u||^{2}+2 F_{f}(u)\right)=0
$$

and

$$
d \phi=d\left(|d u|^{p / 2}\right)=0 .
$$

$d \phi=0$ means $|d u|=$ const. Suppose $|d u|=C>0$. Then the finiteness of the $(p, f)$-energy $E_{p, f}(u)$ of $u$ gives that $\operatorname{Vol}_{f}(M)<+\infty$. If either $|f|$ is uniformly bounded or $f$ is convex and the set of its critical points is unbounded, then Theorems 1.3 and 5.3 in [12] implies that $M$ has at least linear $f$-volume growth, giving a contradiction.

The Kato's inequality with equality holding means $\nabla d u \equiv 0$, i.e. $u: M \rightarrow N$ is totally geodesic, which in turn gives that $u$ is harmonic, i.e. $\tau(u)=0$. Since $|d u|=$ const, $\tau_{p, f}(u)=0$ and $\tau(u)=0$, (2.1) becomes

$$
|d u|^{p-2} d u(\nabla f)=e^{f} \tau_{p, f}(u)-|d u|^{p-2} \tau(u)=0 .
$$

Accordingly, the (3.1) reads

$$
|d u|^{p-2} F_{f}(u)=0,
$$

and by the curvature sign assumptions both

$$
|d u|^{p-2} \sum\left\langle d u\left(\operatorname{Ric}_{f}^{M} e_{i}\right), d u\left(e_{i}\right)\right\rangle=0,
$$

and

$$
|d u|^{p-2} \sum\left\langle R^{N}\left(d u\left(e_{i}\right), d u\left(e_{j}\right)\right) d u\left(e_{i}\right), d u\left(e_{j}\right)\right\rangle=0 .
$$

First, suppose that $|d u|=C>0$ and $\operatorname{Sect}^{N}<0$, then $d u\left(E_{i}\right) \| d u\left(E_{j}\right)$ for all $i, j=1, \ldots, n$ and we conclude that $u(M)$ must be contained in a geodesic of $N$.

On the other hand, suppose that $\left.\operatorname{Ric}_{f}^{M}\right|_{q_{0}}>0$ at some point $q_{0} \in M$. Then necessarily $d u\left(q_{0}\right)=0$ which gives $d u \equiv 0$.

Moreover, (3.5) can be rewrote

$$
|d u|^{p-2}\left(\sum\left\langle d u\left(\operatorname{Ric}^{M} e_{i}\right), d u\left(e_{i}\right)\right\rangle+\left\langle d u\left(\nabla_{e_{i}} \nabla f\right), d u\left(e_{i}\right)\right\rangle\right)=0 .
$$


Since

$$
0=(\nabla d u)(X, Y)=\left(\nabla_{d u(Y)} d u\right)(X)=\nabla_{d u(Y)}^{N} d u(X)-d u\left(\nabla_{Y}^{M} X\right)
$$

for all $X, Y$ vector fields on $M$. (3.3) implies

$$
|d u|^{p-2}\left\langle d u\left(\nabla_{e_{i}}^{M} \nabla f\right), d u\left(e_{i}\right)\right\rangle=|d u|^{p-2}\left\langle\nabla_{d u\left(e_{i}\right)}^{N} d u(\nabla f), d u\left(e_{i}\right)\right\rangle=0,
$$

for each $i=1, \ldots, n$. Since $\operatorname{Ric}_{f}^{M} \geq 0$. (3.7) in particular gives

$$
|d u|^{p-2} \sum\left\langle d u\left(\operatorname{Ric}^{M} e_{i}\right), d u\left(e_{i}\right)\right\rangle=0 .
$$

Hence, if there exists a point $q_{1} \in M$ such that $\left.\operatorname{Ric}^{M}(X, X)\right|_{q_{1}} \neq 0$ for all $0 \neq X \in T_{q_{1}} M$, then $u$ is once again necessarily constant.

\section{A remark}

When $M$ is an $n$-dimensional compact Riemannian manifold without boundary, we have the following result, which is an extension of facts in $p$-harmonic map case. (See Eells and Sampson [4].)

THEOREM 4.1. Let $\left(M^{m}, g, e^{-f} d V_{M}\right)$ be a weighted, compact Riemannian manifold without boundary and $N$ be a smooth Riemannian manifold, and $u: M \rightarrow N$ be a $(p, f)$-harmonic map.

(a) Assume $\operatorname{Ric}_{f}^{M} \geq 0$ and $\operatorname{Sect}^{N} \leq 0$. Then $u$ is totally geodesic.

(b) In addition to (a), if $\operatorname{Ric}_{f}^{M}>0$ somewhere, then $u$ is a constant map.

(c) In addition to (a), if $\operatorname{Sect}^{N}<0$, then $u$ is a constant map, or $u$ maps onto a closed geodesic in $N$.

Proof. Using the Lemma 2.2, we get

$$
\begin{aligned}
0= & \int_{M} L_{f}\left(\frac{1}{p}|d u|^{p}\right) e^{-f} d v_{g} \\
= & \int_{M}|d u|^{p-2}\left(|\nabla d u|^{2}+(p-2)|\nabla| d u||^{2}+F_{f}(u)\right) e^{-f} d v_{g} \\
& +\int_{M}|d u|^{p-2}\langle d(\tau(u)-d u(\nabla f)), d u\rangle e^{-f} d v_{g} \\
= & \int_{M}|d u|^{p-2}\left(|\nabla d u|^{2}+(p-2)|\nabla| d u||^{2}+F_{f}(u)\right) e^{-f} d v_{g} \\
& +\int_{M}\left\langle d(\tau(u)-d u(\nabla f)), e^{-f}|d u|^{p-2} d u\right\rangle d v_{g}
\end{aligned}
$$




$$
\begin{aligned}
= & \int_{M}|d u|^{p-2}\left(|\nabla d u|^{2}+(p-2)|\nabla| d u||^{2}+F_{f}(u)\right) e^{-f} d v_{g} \\
& +\int_{M}\left\langle\tau(u)-d u(\nabla f), \delta\left(e^{-f}|d u|^{p-2} d u\right)\right\rangle d v_{g} \\
= & \int_{M}|d u|^{p-2}\left(|\nabla d u|^{2}+(p-2)|\nabla| d u||^{2}+F_{f}(u)\right) e^{-f} d v_{g} \\
& +\int_{M}\left\langle\tau(u)-d u(\nabla f), \tau_{p, f}(u)\right\rangle d v_{g}
\end{aligned}
$$

Since $u: M \rightarrow N$ be a $(p, f)$-harmonic map, that is, $\tau_{p, f}(u)=0$, we know

$$
\int_{M}|d u|^{p-2}\left(|\nabla d u|^{2}+(p-2)|\nabla| d u||^{2}+F_{f}(u)\right) e^{-f} d v_{g} \leq 0,
$$

By the curvature sign assumptions, we know $F_{f}(u) \geq 0$, and

$$
|d u|^{p-2}\left(|\nabla d u|^{2}+(p-2)|\nabla| d u||^{2}+F_{f}(u)\right) \geq 0 .
$$

Hence

$$
|d u|^{p-2}\left(|\nabla d u|^{2}+(p-2)|\nabla| d u||^{2}+F_{f}(u)\right)=0 .
$$

Analysis similar to that in the proof of Theorem 1.3 shows that the theorem holds.

\section{REFERENCES}

[ 1 ] D. BAKRY AND M. Émery, Diffusions hypercontractives, Séminaire de probabilités XIX, Lect. notes math. 1123, 1983/1984, 177-206 (in French).

[2] P. Baird And S. Gudmundsson, p-harmonic maps and minimal submanifolds, Math. Ann. 294 (1992), 611-624.

[ 3 ] J. Eells and L. Lemaire, Selected topics in harmonic maps, CBMS Reg. Conf. Ser. Math. 50 (1983), published for the Conference Board of the Mathematical Sciences, Washington, D.C.

[ 4 ] J. Eells And J. H. SAmpson, Harmonic mappings of Riemannian manifolds, Amer. J. Math. 86 (1964), 109-160.

[5] J. Eells and L. Lemaire, A report on harmonic maps, Bull. Lond. Math. Soc. 10 (1978), $1-68$.

[6] A. Lichnerowicz, Applications harmoniques et variétés kähleriennes, Symposia Mathematica III (INDAM, Rome, 1968/69), Academic Press, London, 1968/69, 341-402 (in French).

[7] A. Lichnerowicz, Variétés riemanniennes à tenseur C non négatif, C.R. Acad. Sci. Paris, Sér. A-B 271 (1970), A650-A653 (in French).

[8] D. J. Moon, H. L. LiU AND S. D. Jung, Liouville type theorems for $p$-harmonic maps, J. Math. Anal. Appl. 342 (2008), 354-360.

[ 9 ] N. NaKauchi, A Liouville type theorem for $p$-harmonic maps, Osaka J. of Math. 35 (1998), 303-312.

[10] M. Rimoldia and G. Veronellib, Topology of steady and expanding gradient Ricci solitons via $f$-harmonic maps, Differential Geometry and its Applications 31 (2013), 623-638. 
[11] R. M. Schoen AND S. T. YAU, Harmonic maps and the topology of stable hypersurfaces and manifolds of nonnegative Ricci curvature, Comm. Math. Helv. 51 (1976), 333-341.

[12] G. Wei AND W. Wylie, Comparison geometry for the Bakry-Emery Ricci tensor, J. Differ. Geom. 83 (2009), 377-405.

\author{
Jian-Feng Zhang \\ Department of Mathematics \\ LISHUI UNIVERSITY \\ Lishui, ZHEJIANG 323000 \\ P.R. CHINA \\ E-mail: zjf7212@163.com \\ Yue Wang \\ Department of Mathematics \\ ChINA JiLIANG UNIVERSITY \\ HANGZHOU 310018 \\ P.R. CHINA \\ E-mail: kellywong@cjlu.edu.cn
}

